Meta

Journal des traducteurs

Translators' Journal

\title{
Réflexions sur la formation générale du traducteur
}

\section{Jean Darbelnet}

Volume 11, numéro 4, décembre 1966

La formation du traducteur

URI : https://id.erudit.org/iderudit/002467ar

DOI : https://doi.org/10.7202/002467ar

Aller au sommaire du numéro

Éditeur(s)

Les Presses de l'Université de Montréal

ISSN

0026-0452 (imprimé)

1492-1421 (numérique)

Découvrir la revue

Citer cet article

Darbelnet, J. (1966). Réflexions sur la formation générale du traducteur. Meta, 11(4), 155-160. https://doi.org/10.7202/002467ar d'utilisation que vous pouvez consulter en ligne.

https://apropos.erudit.org/fr/usagers/politique-dutilisation/ 


\section{RÉFLEXIONS SUR \\ LA \\ FORMATION GÉNÉRALE \\ DU \\ TRADUCTEUR}

À LA SUITE du deuxième congrès des traducteurs et interprètes du Canada, en avril 1963, le Journal des Traducteurs a publié dans son numéro d'octobredécembre de la même année le texte des remarques que j'avais faites, à l'une des séances, sur la formation du traducteur. L'occasion m'est donnée aujourd'hui de reprendre ce sujet en l'élargissant.

Il ne sera question ici que de la formation générale du traducteur, c'est-àdire de la formation qu'il doit recevoir en dehors de toute spécialisation. Peutêtre n'est-il pas inutile, pour commencer, de justifier cette distinction qui n'est sans doute pas évidente pour tout le monde.

Il est généralement reconnu que celui qui traduit des textes techniques doit se familiariser avec le vocabulaire des sujets traités dans ces textes, et, par conséquent, dans une certaine mesure tout au moins, avec les choses que les mots représentent. Cela explique qu'il doive se spécialiser assez étroitement, le nombre de terminologies que peut assimiler un seul individu étant limité. Pour beaucoup de traducteurs les problèmes de traduction sont surtout des problèmes de termes techniques, l'insertion de ces termes dans un texte étant relativement facile, du moins c'est ce qu'on pense. Et l'exactitude terminologique est pour le traducteur une préoccupation légitime. Mais elle ne doit pas être la seule. Ce qu'on comprend moins généralement, c'est que l'entourage des termes techniques, qui est en quelque sorte la chaîne du texte, dont ces termes forment la trame, a aussi une grande importance. Le message que l'énoncé est censé transmettre dépend pour sa clarté et de l'exactitude terminologique et de la façon dont il est composé.

Ces considérations justifient, il me semble, la nécessité pour les traducteurs non seulement de bien posséder le vocabulaire technique, mais encore d'apprendre à mieux connaître leur instrument, c'est-à-dire la langue dans laquelle ils traduisent. Cela étant admis, il vaut la peine de se demander quelle doit être la nature de l'enseignement qu'ils doivent recevoir pour atteindre ce deuxième objectif.

Tout d'abord il importe de se rendre compte que, dans un pays bilingue comme le Canada, le rôle du traducteur est double. Il doit, comme partout ailleurs, renseigner exactement son lecteur sur le contenu d'un texte rédigé dans une autre langue. Il doit aussi protéger la langue maternelle contre les infiltra- 
tions de l'autre langue officielle. On ne saurait exagérer l'importance de ce second rôle. Qu'on le veuille ou non, dans un pays bilingue la langue minoritaire est une langue de traduction. On peut éviter qu'elle le soit trop, on ne peut faire en sorte qu'elle ne le soit pas du tout. De la qualité de la traduction dépend en partie - en grande partie - celle de la langue qu'on parle et écrit. Disons donc que le traducteur est à la fois au service du public et au service de la langue.

Pour s'acquitter de cette seconde obligation, il doit bien connaître la langue qu'il a pour mission de servir et de protéger, et nous reviendrons tout à l'heure sur ce point. Mais aussi bien pour traduire exactement que pour bien rédiger, il lui faut respecter un principe essentiel, à savoir que la valeur d'une traduction ne dépend pas de sa littéralité. Par ce terme il faut entendre l'abus de la traduction littérale, et par littéralisme, la tendance à la littéralité. Nous ne parlerons pas de littéralité ou de littéralisme quand la bonne traduction se trouve être littérale. De ce danger qu'est la traduction littérale intempestive, le traducteur doit être convaincu au point de pouvoir à son tour convaincre son employeur. Le public a en effet trop tendance à croire que la traduction est d'autant plus exacte qu'elle épouse la contexture de l'original. On ne médite pas assez cette remarque d'Hilaire Belloc que le traducteur doit s'émanciper de deux servitudes, celle de la longueur et celle de la forme. Cela veut dire qu'il est libre de rendre un passif par un actif, de changer l'ordre des mots, d'allonger ou de raccourcir les phrases, du moment qu'il rend exactement le sens de l'original. C'est cette idée qui devrait être au centre d'un cours de traduction générale dans lequel on apprend à utiliser les ressources de la langue d'arrivée sans forcer son génie. C'est le rôle d'un cours de ce genre de montrer par des exercices précis que la traduction littérale est la meilleure seulement quand elle respecte a) le sens du message, b) sa valeur stylistique, c) sa métalinguistique, d) le génie de la langue d'arrivée. Le mot génie n'est guère en faveur auprès des linguistes d'aujourd'hui, qui lui reprochent d'être trop subjectif. Mais il est commode pour désigner l'ensemble des démarches de la langue, c'est-à-dire des combinaisons de mots que la langue préfère, encore que d'autres soient possibles, en somme de ce qu'on pourrait appeler ses constructions idiomatiques, ce qui est idiomatique se situant au-delà de ce qui est purement grammatical.

Il arrive justement que beaucoup de traductions respectent la grammaire mais non le génie de la langue d'arrivée. Elles sont correctes, mais non idiomatiques. On peut facilement en donner des exemples, exemples de mots et exemples de phrases. Et puisque nous considérons surtout le passage de l'anglais au français, nous pouvons prévoir que c'est à l'anglicisme qu'aboutit la littéralité dans ce domaine.

Les anglicismes, on le sait, sont nombreux et variés. Et parmi les différentes sortes, il en est une qui est celle des anglicismes de traduction. Sans doute tous les anglicismes sont-ils dus à des traductions fautives, mais ils le sont plus ou moins directement, et il en est qui révèlent plus que d'autres l'embarras, la gaucherie du traducteur qui s'est laissé imposer, pour traduire, le mot le plus proche de l'anglais ou encore celui que le dictionnaire donne comme une traduction passe-partout du terme anglais correspondant. Sans tenter de traiter cette question à fond, il est bon de donner quelques échantillons, car il s'agit d'une tendance néfaste, que les traducteurs doivent apprendre à combattre. 
Ainsi on parle des «octrois » fédéraux sans doute parce que to grant peut vouloir dire octroyer, et qu'on est passé du verbe au substantif. On a ainsi généralisé une traduction qui ne vaut que dans certains cas et on l'a fait aux dépens de subventions. Le même processus est reconnaissable à propos de « maîtriser » qui semble rendre parfaitement to master. Mais la similitude de forme n'entraîne pas forcément l'identité de sens ou de valeur. Il est exact que to hit signifie frapper, mais nous sommes heurtés et non pas «frappés» par une autre voiture. Quand je lis qu' « un chauffard nous a frappés», je vois le chauffard descendre de sa voiture pour se livrer à des voies de fait sur d'autres automobilistes. Toujours à propos d'accidents de la circulation, les journaux disent que telle personne a été blessée alors qu'elle «voyageait» dans la voiture de $\mathrm{M}$. X. Ce verbe «voyager» nous vient de to ride qu'il aurait fallu rendre par avoir pris place, se trouver. Quelquefois c'est d'un accident d'aviation qu'il s'agit, et on nous parle d' «écrasement» (a crash). Or, si le verbe s'écraser peut se dire d'un avion, on ne parle pas de l' « écrasement» d'un avion. Il faut nous contenter en français d'un terme moins évocateur. On dit tout simplement un accident d'aviation là où l'anglais dit air crash ou plane crash.

Pourquoi voit-on l'inscription «air climatisé » alors que climatisé suffirait, puisqu'on ne peut climatiser un local qu'en climatisant l'air qui y circule. Il y a fort à parier que le mot « air »n'est là qu'à cause de air-conditioned. Il se trouve qu'en anglais conditioned a besoin d'être modifié par air. Climatisé peut se passer de cette précision.

C'est encore au littéralisme que nous devons «véhicules moteurs » pour véhicules automobiles, et «chambre avec bain» pour chambre avec salle de bain. Parfois le mécanisme fautif de la traduction est moins apparent. Si on voit « déménager » suivi de la nouvelle adresse, c'est parce qu'on traduit mécaniquement to move par «déménager». Ici encore nous nous trouvons en présence d'une généralisation abusive, comme dans le cas de to grant. Il est exact que to move a bien ce sens dans «We are moving next week». Mais on ne déménage pas à un endroit, on y emménage, et dans les textes commerciaux c'est transférer qui indique le mieux le changement d'adresse d'un établissement. Plus subtil est le cas de la foudre qui «frappe» une étable (lightning strikes ...) au lieu de tombe sur...

Il y a quelque temps, parlant à une émission de la Parole est d'or, M. Vinay a regretté, avec raison, qu'on n'accepte pas sans profession comme équivalent de housewife dans les recensements et les statistiques. Je crois que c'est justement parce que le public et certains traducteurs répugnent à employer un équivalent qui n'ait pas la même forme que l'expression à traduire. On persiste à vouloir rendre un nom par un nom et une locution par une locution. On a l'impression qu'une traduction de structure différente ne peut pas être exacte. D'où la préférence pour ménagère qui a la même fonction grammaticale que housewife et aussi l'un de ses sens, mais, par contre, n'a pas cours dans la langue administrative. C'est un bon exemple de la tyrannie de la forme. L'esprit humain préfère se laisser guider par la forme, plutôt que de voir ce qu'il y a derrière. La tyrannie de la forme, c'est la tyrannie des apparences. Depuis longtemps les philosophes dénoncent ce qu'il y a de trompeur dans les apparences. Le moment est venu d'en faire l'application à la traduction. 
C'est de ce genre d'erreur qu'il faut préserver l'apprenti traducteur et c'est dans un cours de traduction générale qu'on peut le plus facilement l'habituer à reconnaître la diversité des sens sous le parallélisme des formes et la diversité des formes correspondant à une identité de sens. Bien entendu, on signalera au passage les cas où la traduction littérale est satisfaisante à tous points de vue, ce qui n'est pas tellement rare. L'essentiel est de montrer qu'il faut passer par l'idée avant d'aboutir à la traduction, de mettre en quelque sorte les mots au service de la pensée, et non de les asservir à d'autres mots. Il y a là tout un travail d'endoctrination qu'il est nécessaire d'accomplir, si l'on veut que les traducteurs comprennent vraiment leur métier et le fassent comprendre aux autres.

Il est logique de commencer par des mots comme ceux qui ont été examinés plus haut, et de passer ensuite à des phrases dont les équivalents exacts sont de structure différente. On pourra ainsi illustrer le principe que bien souvent il faut changer de structure pour éviter de changer le sens. Et pour aborder l'étude des phrases, il est sans doute préférable de donner en même temps la traduction et de faire faire une analyse du texte anglais et du texte français de façon à bien montrer que le second a exactement le même sens que le premier, et cela malgré le fait que les mots pris séparément ne se traduisent pas l'un par l'autre et qu'ils sont, dans chaque texte, agencés différemment. Cette analyse peut se faire au moyen d'une technique de découpage et de numérotage des éléments ainsi dégagés, dont M. Vinay et moi avons donné des exemples dans notre Stylistique comparée du français et de l'anglais. Ainsi on peut démontrer que pour des raisons indépendantes de notre volonté traduit exactement for reasons beyond our control, et que faire preuve de mauvaise volonté peut, dans certains cas, correspondre à to be unco-operative.

En somme, à côté de la transposition grammaticale qui fait permuter les parties du discours d'une langue à l'autre au nom de leur identité de sens, il y a une transposition des mots qui fait que co-operation dans une langue évoque la bonne volonté dans l'autre. Cela n'empêche pas que dans certains cas co-operation corresponde à... coopération.

Pour résumer cette partie de mon propos, on peut dire que la traduction littérale à jet continu déforme la langue d'arrivée. Il arrive aussi qu'elle gêne la compréhension. Et à ce sujet il importe de montrer, avec exemples à l'appui, que le mot à mot peut aboutir à un contresens. C'est le cas de he is sure to come traduit par il est sûr de venir. Si ce mode de traduction est encore si largement pratiqué, cela tient sans doute à deux raisons. D'une part le littéralisme bénéficie d'un préjugé tenace, et d'autre part les traducteurs sont le plus souvent obligés de travailler vite. Or, la traduction littérale prend moins de temps que celle que nous recommandons, qui oblige à passer par l'idée avant de choisir les mots de la langue d'arrivée. L'ignorance, ou plutôt une imparfaite compréhension des processus de la traduction, et la hâte sont les deux ennemis de la qualité dans ce domaine. Cela il faut l'enseigner, textes en mains, et en proposant aux élèves des exercices courts, précis et gradués.

Lorsqu'en 1943 je fus chargé par le colonel Bovey d'organiser les cours de traduction à l'Université McGill conjointement avec la Société des Traducteurs de Montréal (comme elle s'appelait alors), j'avais prévu un examen d'entrée, des 
exercices de traduction dans les deux sens pendant l'année et un examen de fin d'année. Nous tenions à ce que les anglophones sachent suffisamment le français et à ce que les francophones sachent suffisamment l'anglais. En d'autres termes, nous voulions nous assurer que chacun avait une bonne connaissance de la langue seconde. Nous admettions d'emblée que les étudiants possédaient bien leur langue maternelle et qu'au surplus les exercices faits au cours de l'année permettraient de rectifier certaines habitudes et d'étendre ou de préciser ce qu'ils savaient déjà.

Depuis, mon expérience et mes réflexions m'ont fait voir la question différemment. Tout ce qui vient d'être décrit ci-dessus reste valable, mais ne suffit pas. Je suis arrivé à cette conclusion que, dans les cours de traduction générale, l'élève doit non seulement apprendre à traduire, mais aussi se perfectionner dans les deux langues en présence et, par conséquent, dans sa langue maternelle. La vérité est qu'on croit savoir cette langue parce qu'on ne se rappelle pas quand on l'a apprise, mais qu'en fait il reste beaucoup à apprendre. L'illusion vient de ce qu'on la parle couramment et sans effort. Dès qu'on est obligé de rédiger, on s'aperçoit qu'on hésite sur beaucoup de points et qu'il est souvent difficile d'exprimer toute sa pensée. Il est exact que notre développement intellectuel, nos études, nos occupations ont pour effet d'élargir le champ de notre activité linguistique. Mais cela est surtout vrai dans le domaine du vocabulaire: nous savons plus de mots parce que nous connaissons plus de choses. Mais il ne s'ensuit pas que la syntaxe y gagne en finesse et en sûreté. L'expérience montre qu'il faut observer les faits de langue et y réfléchir si l'on veut vraiment avoir bien en main cet outil indispensable qu'est la langue maternelle, langue de pensée et non pas seulement de communication.

Comme je le faisais remarquer dans l'article auquel il a déjà été fait allusion, il n'est pas exagéré de dire que le traducteur doit mieux connaître sa langue que l'écrivain. Celui-ci choisit ce qu'il écrit et il lui est loisible de rester à l'intérieur d'étroites limites. On connaît des auteurs qui ont su dire ce qu'ils avaient à dire avec un très petit effectif de mots et de tours. Le traducteur ne choisit pas le sujet à traiter. Quelqu'un l'a déjà choisi pour lui, et il ne sait jamais à quelles ressources de la langue d'arrivée il devra faire appel pour rendre une pensée qu'il n'a pas conduite à sa guise mais qu'il reçoit toute faite.

Dans ces conditions, il semble bien que ce soit le rôle d'un enseignement comme celui que nous envisageons d'aider les élèves, non seulement à se familiariser avec les mécanismes de la traduction, mais aussi à parfaire leur connaissance des deux langues qu'ils utilisent, à la fois à propos et en dehors des textes qu'ils ont à traduire. Cela suppose un enseignement parallèle à celui de la traduction proprement dite.

Sans doute, il ne saurait être question de faire des cours séparés de grammaire et de lexicologie qui absorberaient une part excessive de l'horaire dont on dispose. Mais au lieu de se contenter de corriger les erreurs ou les insuffisances d'une traduction il conviendrait, à propos du texte, de faire le point sur les questions de grammaire et de vocabulaire qui se trouvent ainsi soulevées. Cela suppose que le professeur, en corrigeant ses copies, a réfléchi aux causes des fautes commises. Celles-ci peuvent évidemment tenir à ce que le texte a été mal compris. Mais nous pensons plutôt ici aux déficiences d'ordre grammatical ou lexical dans 
le domaine de la langue d'arrivée. Il y a une lacune à combler, et pour ce faire il importe de reprendre par la base le fait de grammaire ou de vocabulaire qui est à l'origine de l'erreur relevée. Pareille façon de procéder suppose de la part du professeur une conception particulière de son enseignement. Il doit choisir entre la qualité et la quantité, et il doit accepter de faire moins, d'aller moins vite, pour faire les choses plus à fond.

S'il est difficile de faire revoir les questions de grammaire et de vocabulaire dans des cours auxiliaires distincts du cours de traduction proprement dit, on pourrait cependant envisager de consacrer une partie du temps à des explications de textes conçues en vue de la traduction. Il ne saurait s'agir d'analyse littéraire, et il $\mathrm{y}$ aurait évidemment à déterminer un nouveau mode d'explication où le texte étudié serait considéré comme susceptible de fournir de précieuses indications sur l'emploi des mots et des tours. À l'occasion, on peut utiliser ce qu'on pourrait appeler des textes à trous, c'est-à-dire des textes incomplets et où les vides doivent être comblés au moyen de définitions des mots qui manquent. II faut en effet considérer que le traducteur est obligé de mobiliser très vite les mots qu'il sait mais auxquels il ne pense pas toujours. Tout exercice de mobilisation est donc particulièrement indiqué.

L'utilisation des textes peut donner lieu à un autre genre d'étude. De plus en plus on se rend compte que tout traducteur consciencieux se doit de prendre des notes à propos des mots qu'il rencontre et de faire des dépouillements. Autant qu'il apprenne le plus tôt possible à exploiter un texte, à relever les mots qui servent à rendre tout ce qui se rapporte au sujet particulier qui y est traité. On pense d'abord aux termes techniques, mais on peut concevoir d'autres dépouillements, par exemple celui des charnières ou articulations, de ce qui constitue la dialectique du texte.

Soit à propos des exercices de traduction, soit sous forme d'exposés distincts il y aurait également lieu, surtout dans un pays comme le Canada, de passer en revue les différentes formes d'anglicisation du français. L'avantage de cette étude serait d'affiner chez les élèves le sens de l'anglais tout en les aidant à se protéger contre les anglicismes. L'expérience montre en effet qu'à force de vivre au contact d'une autre langue on perd, si on n'y prend pas garde, la faculté de distinguer entre le français authentique et le français contaminé. La prise de conscience se révèle comme un remède souverain contre les anglicismes insidieux. Une fois qu'un élève a bien saisi la différence entre supporter et to support, entre agenda en français et agenda en anglais, il est peu probable qu'il continue à confondre ces faux amis du traducteur, dont l'étude systématique devrait figurer au programme de tous ceux qui se préparent à la traduction du français en anglais ou vice versa.

On voit par ce qui précède que l'enseignement de la traduction tel qu'il a été conçu depuis qu'il existe, pourrait être modifié de façon à être à la fois plus varié et plus efficace. Cette nouvelle orientation consiste essentiellement à reconnaître que les traducteurs utilisent les ressources des deux langues et qu'ils ne sont pas pleinement préparés tant qu'ils n'ont pas eu l'occasion de réfléchir sur le fonctionnement des langues qu'ils transposent l'une par l'autre.

Jean Darbelnet Québec 